Supporting information

for

\title{
Characterization of Three Tailoring Enzymes in Dutomycin Biosynthesis and Generation of a Potent Antibacterial Analogue
}

Lei Sun, ${ }^{\dagger}, \perp$ Siyuan Wang, ${ }^{\dagger, \perp}$ Shuwei Zhang, ${ }^{\dagger}$ Lei Shao, ${ }^{\dagger}$ Qian Zhang, ${ }^{\dagger}$ Chad Skidmore, ${ }^{\dagger}$ ChengWei Tom Chang, ${ }^{\dagger}$ Dayu Yu, ${ }^{\dagger, \S}$ and Jixun Zhan ${ }^{*} \dagger, \|$

$\dagger^{\dagger}$ Department of Biological Engineering, Utah State University, 4105 Old Main Hill, Logan, UT 84322-4105, USA

$\$$ Department of Chemistry and Biochemistry, Utah State University, 0300 Old Main Hill, Logan, UT 84322-0300, USA

$\S$ Department of Applied Chemistry and Biological Engineering, College of Chemical Engineering, Northeast Dianli University, Jilin, Jilin 132012, China

॥ TCM and Ethnomedicine Innovation \& Development Laboratory, School of Pharmacy, Hunan University of Chinese Medicine, Changsha, Hunan 410208, China

$\perp$ These authors contributed to this work equally.

* Corresponding author: J. Zhan. Tel.: 1-435-797-8774; fax: 1-435-797-1248; e-mail: jixun.zhan@usu.edu 
Table S1. Plasmids used in this study.

\begin{tabular}{ccc}
\hline Plasmid & Description & Reference \\
\hline pLS51 & $k s 1$ (part of $d u t A)$ in pJET1.2 & this work \\
pLS52 & $k s 2$ in pJET1.2 & this work \\
pLS53 & $k s 3$ in pJET1.2 & this work \\
pLS54 & $k s 1$ (part of $d u t A)$ in pKC1139 & this work \\
pLS55 & $k s 2$ in pKC1139 & this work \\
pLS56 & $k s 3$ in pKC1139 & this work \\
pSW70 & $d u t G T 2$ fragment in pJET1.2 & this work \\
pSW75 & $d u t G T 2$ fragment in pKC1139 & this work \\
pSW82 & $d u t M T 1$ fragment in pJET1.2 & this work \\
pSW91 & $d u t M T 1$ fragment in pKC1139 & this work \\
pSW138 & $d u t G T 1$ fragment in pJET1.2 & this work \\
pSW140 & $d u t G T 1$ fragment in pKC1139 & this work \\
pSUN151 & $d u t M T 1$ in pJET1.2 & this work \\
pSUN168 & dutMT1 in pET28a & this work \\
\hline
\end{tabular}


Table S2. Primers used in this study.*

\begin{tabular}{ll}
\hline \multicolumn{1}{c}{ Primer } & \multicolumn{1}{c}{ Sequence } \\
\hline KS $\alpha-F$ & 5'-TSGCSTGCTTCGAYGCSATC-3' \\
KS $\alpha-$ R & 5'-TGGAANCCGCCGAABCCGCT-3' \\
DutMT1-F1 & 5'-AAAAGCTTCCTGCGCGACATGGTCCTGT-3' \\
DutMT1-R1 & 5'-AATCTAGACGACCAGGTTCTCGATCACG-3' \\
DutMT1-F2 & 5'-AACATATGACAGCTCCCGCTCT-3' \\
DutMT1-R2 & 5'-AACTCGAGTCAGTTACCCGTGTCCCGGCGTT-3' \\
DutGT2-F & 5'-AAAAGCTTACGGCATCCTGGACGAGCAT-3' \\
DutGT2-R & 5'-AATCTAGATTCGGCTGGGCGACGATCAA-3' \\
DutGT1-F & 5'-AAAAGCTTGATCCGCGCGCTGTA-3' \\
DutGT1-R & 5'-AATCTAGAATCTCTGTGCAGATCGGTGA -3' \\
DutA-Check1 & 5'-GTCTCCACGGGCTGTACCTC-3' \\
DutA-Check2 & 5'-ATCAGCCGGCTGGGCAGATG-3' \\
DutMT1-Check1 & 5'-ATGACAGCTCCCGCTCTCGAA -3' \\
DutMT1-Check2 & 5'- ATTTATTGGCGTCATCAGGTTGT -3' \\
DutGT2-Check1 & 5'-AGATCAAGCACAGTCCGGA-3' \\
DutGT2-Check2 & 5'-AAGCGACTCTGGAAGAGG-3' \\
DutGT1-Check1 & 5'-ATGGTCGACGAGTACGT-3' \\
DutGT1-Check2 & 5'- TGACGATGTCGATGGCCAA-3' \\
M13-47 & 5'-CGCCAGGGTTTTCCCAGTCACGAC-3' \\
RM-V & 5'-GAGCGGATAACAATTTCACACAGG-3' \\
\hline * Restriction sites are shown in bold.
\end{tabular}




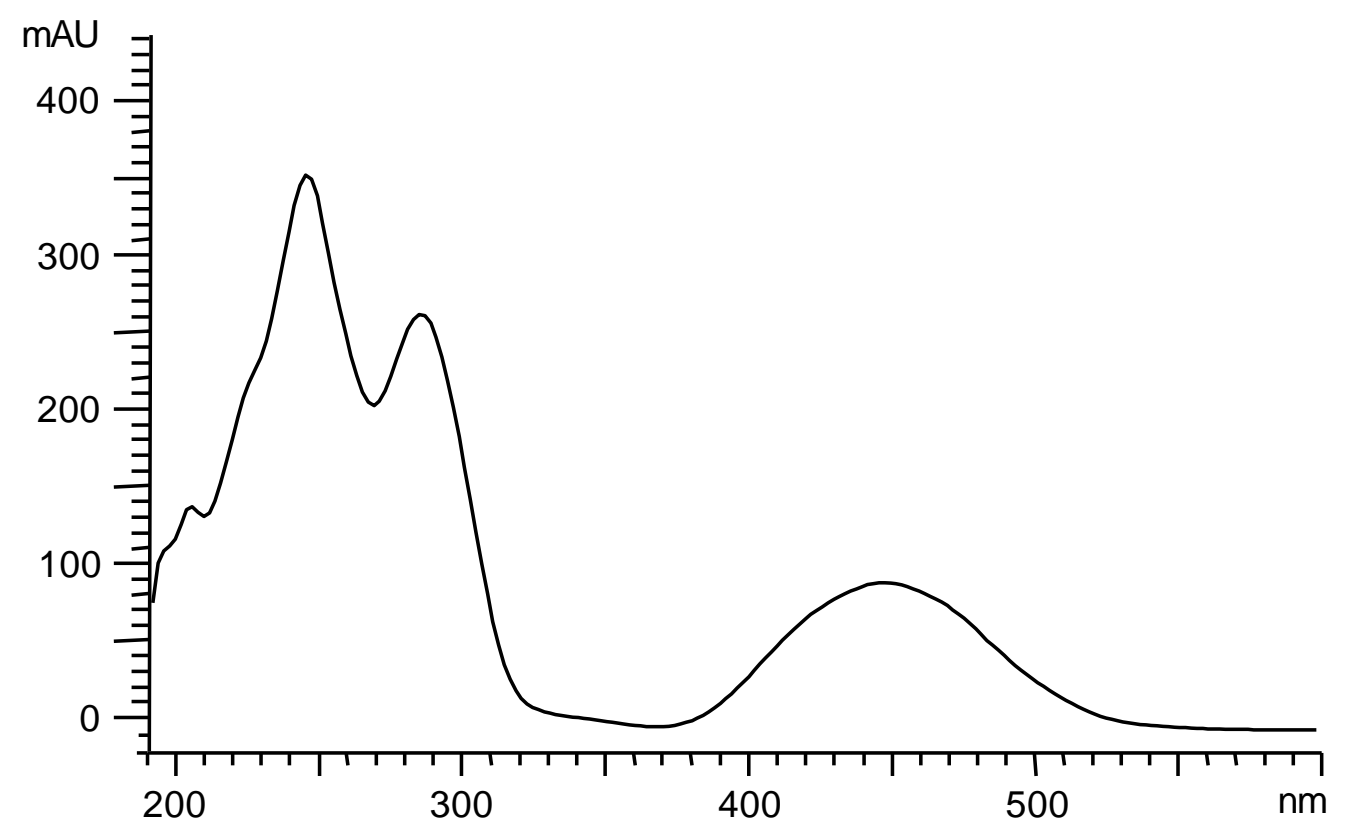

Figure S1. UV spectrum of 1 and 2. 

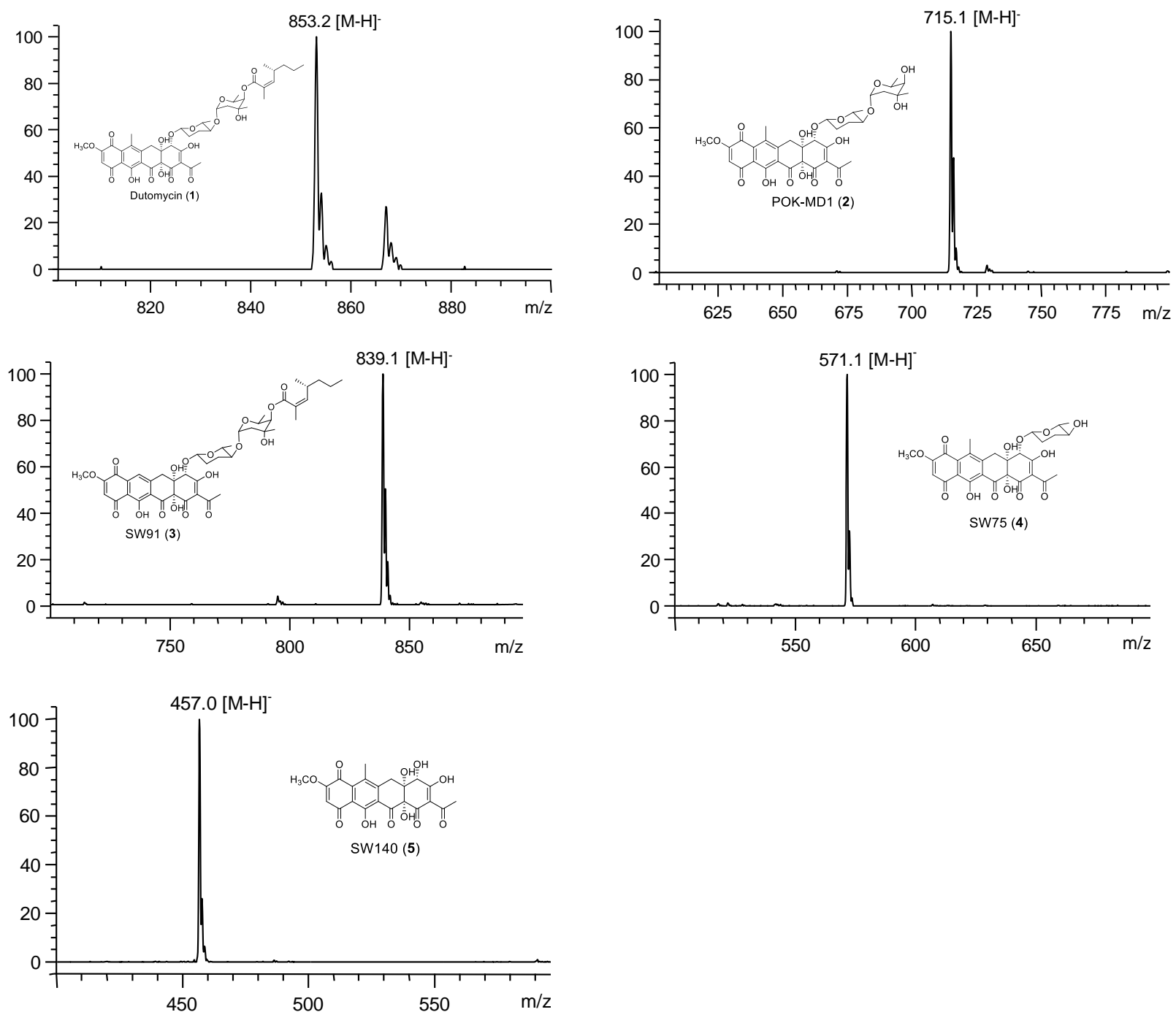

Figure S2. ESI-MS spectra of 1-5. 


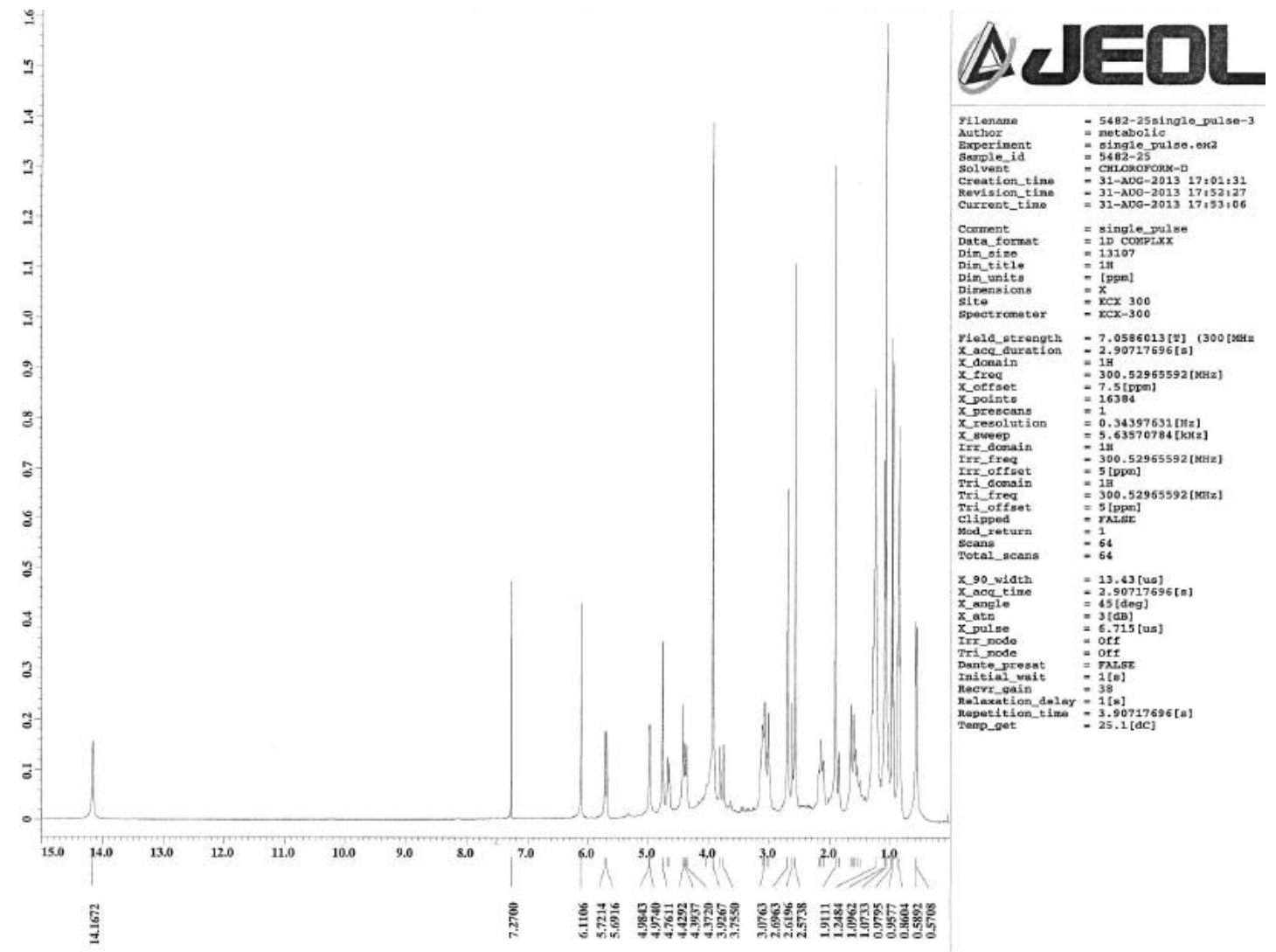

b

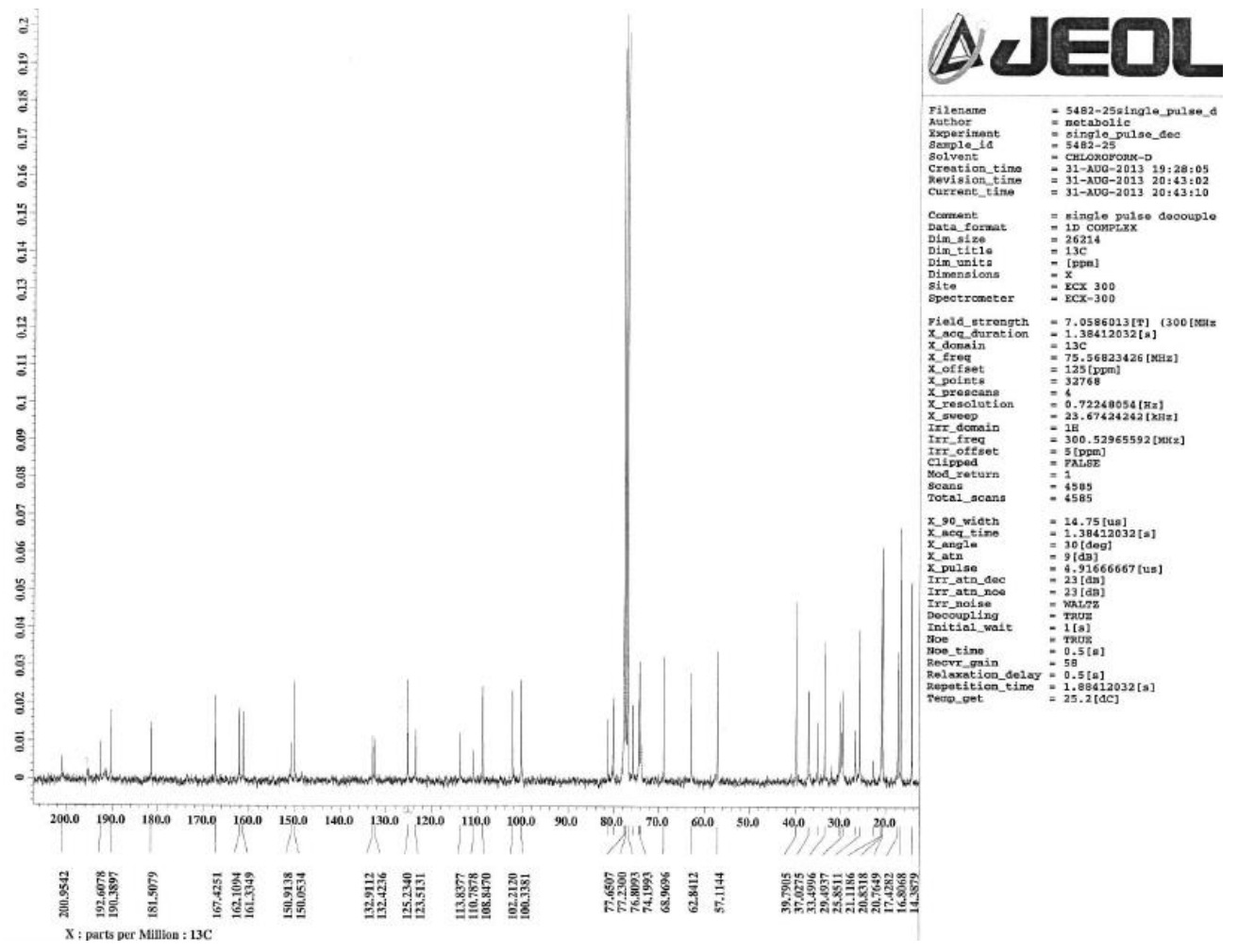


c

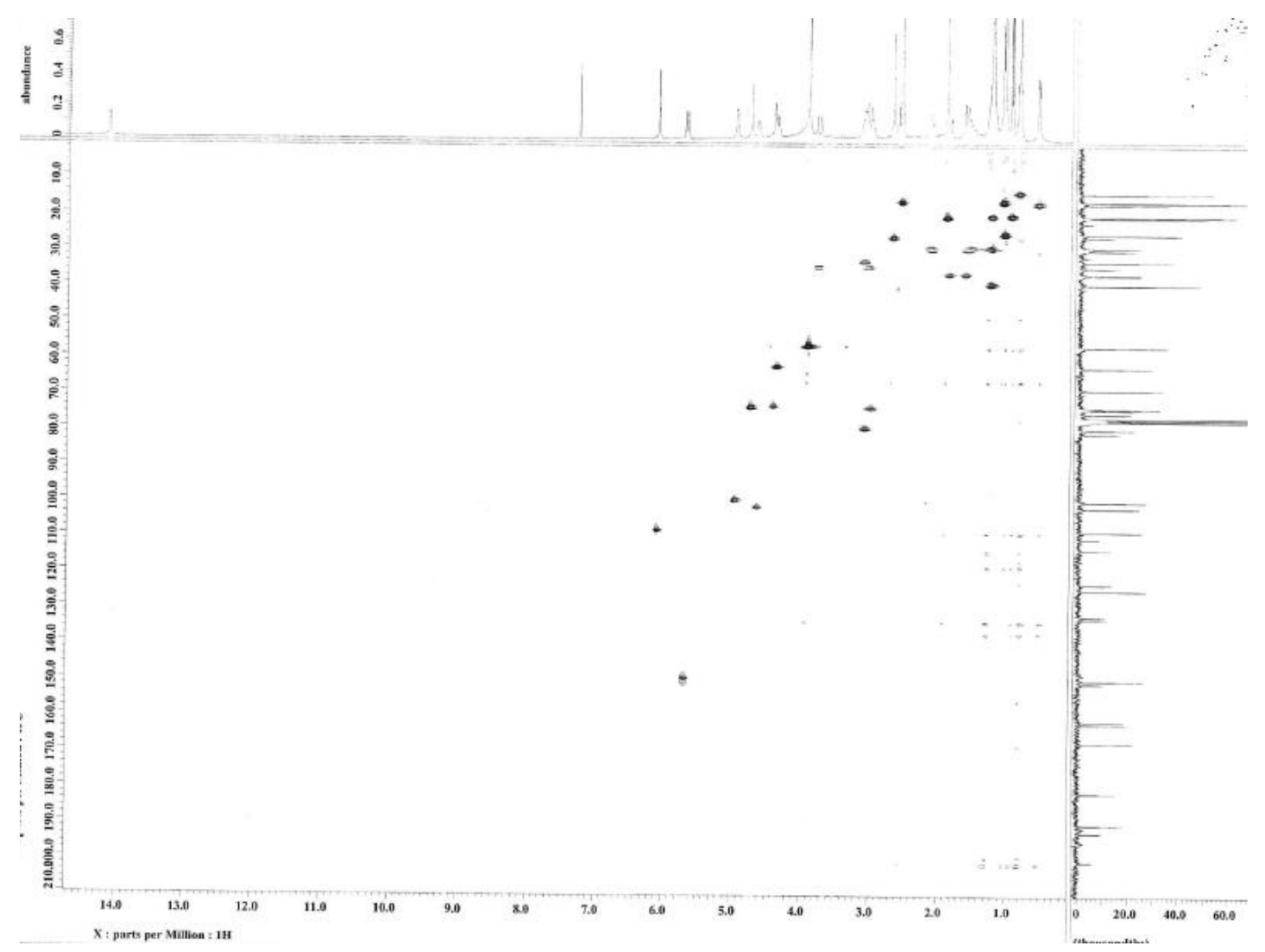

d

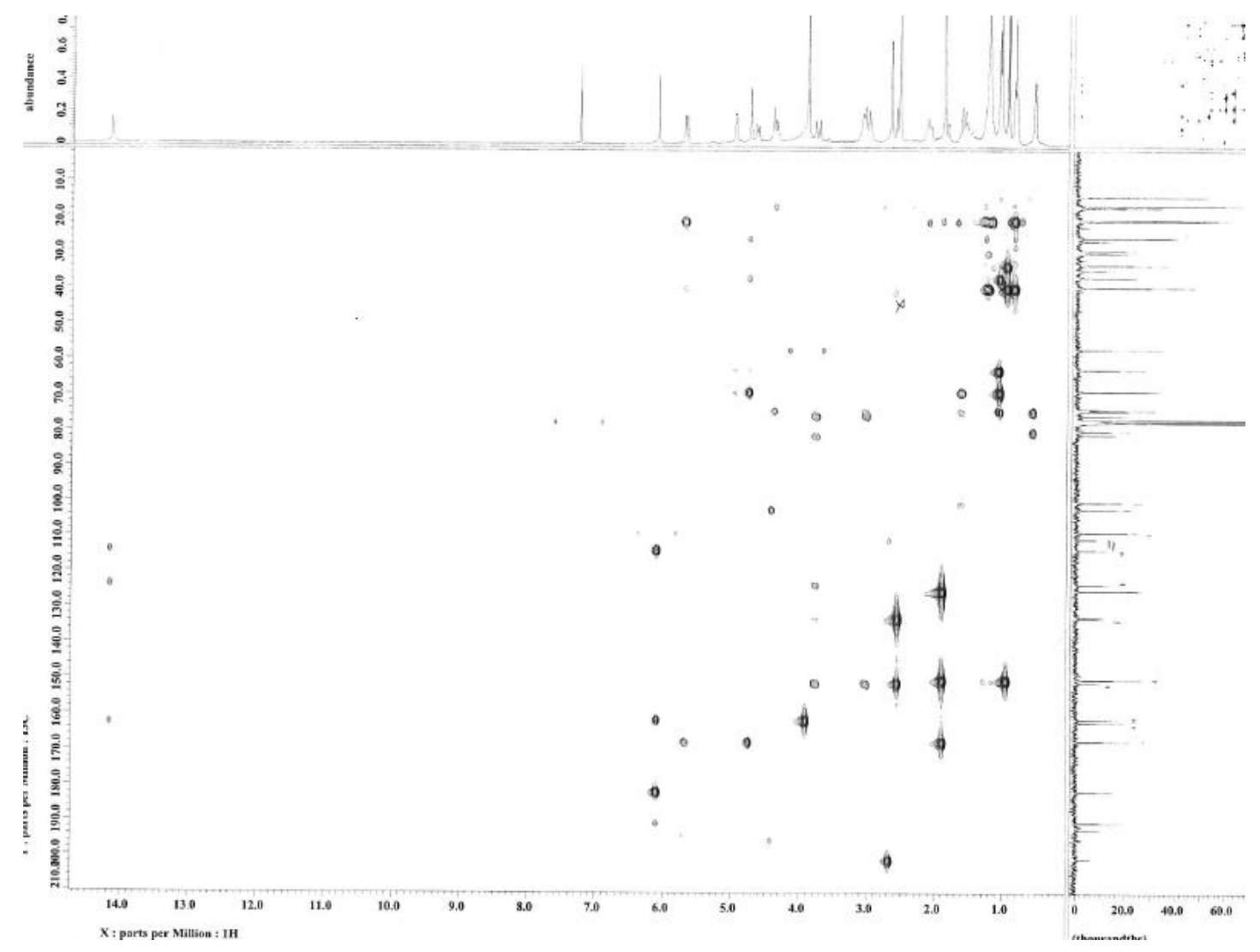


e

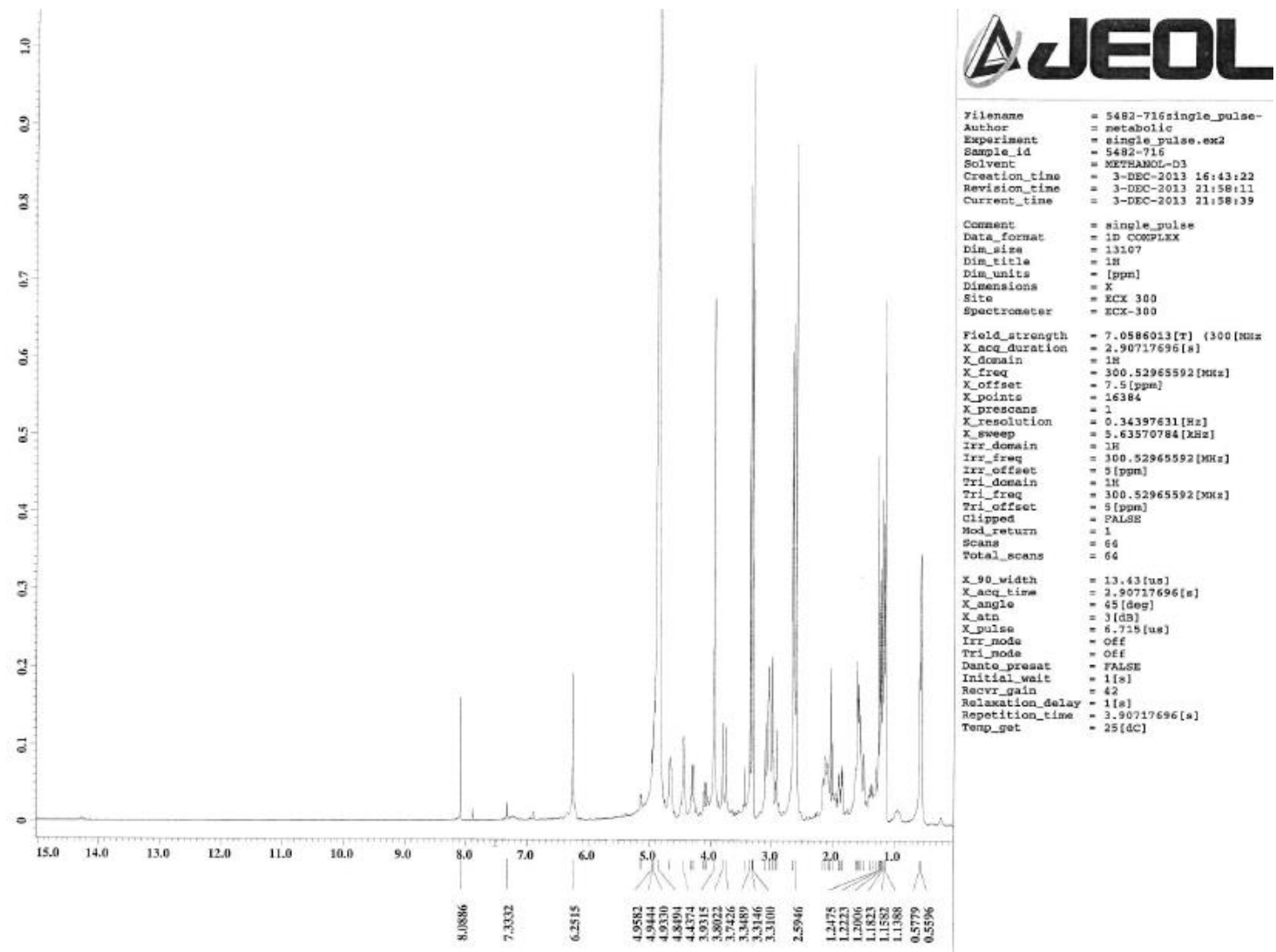

f

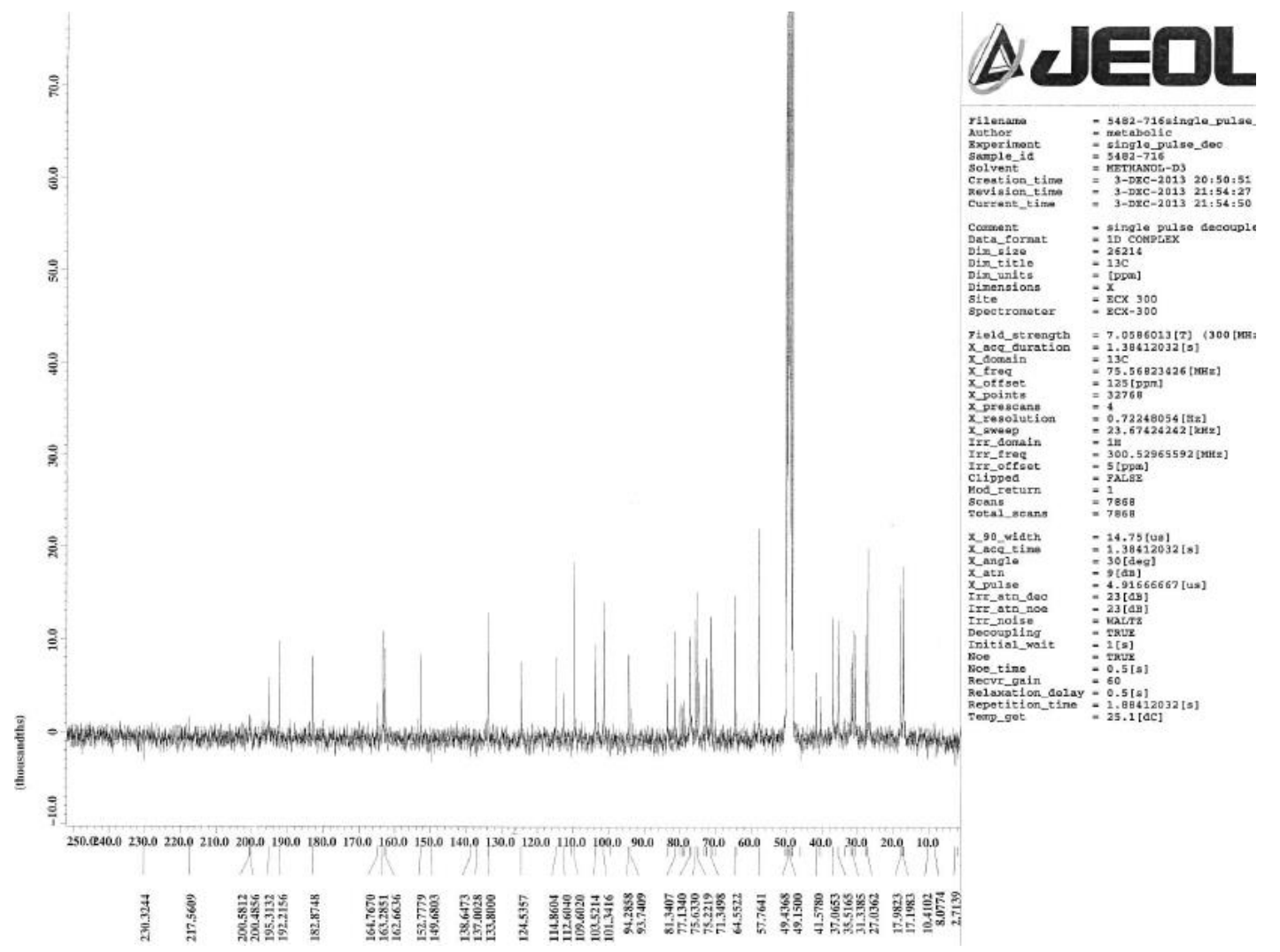




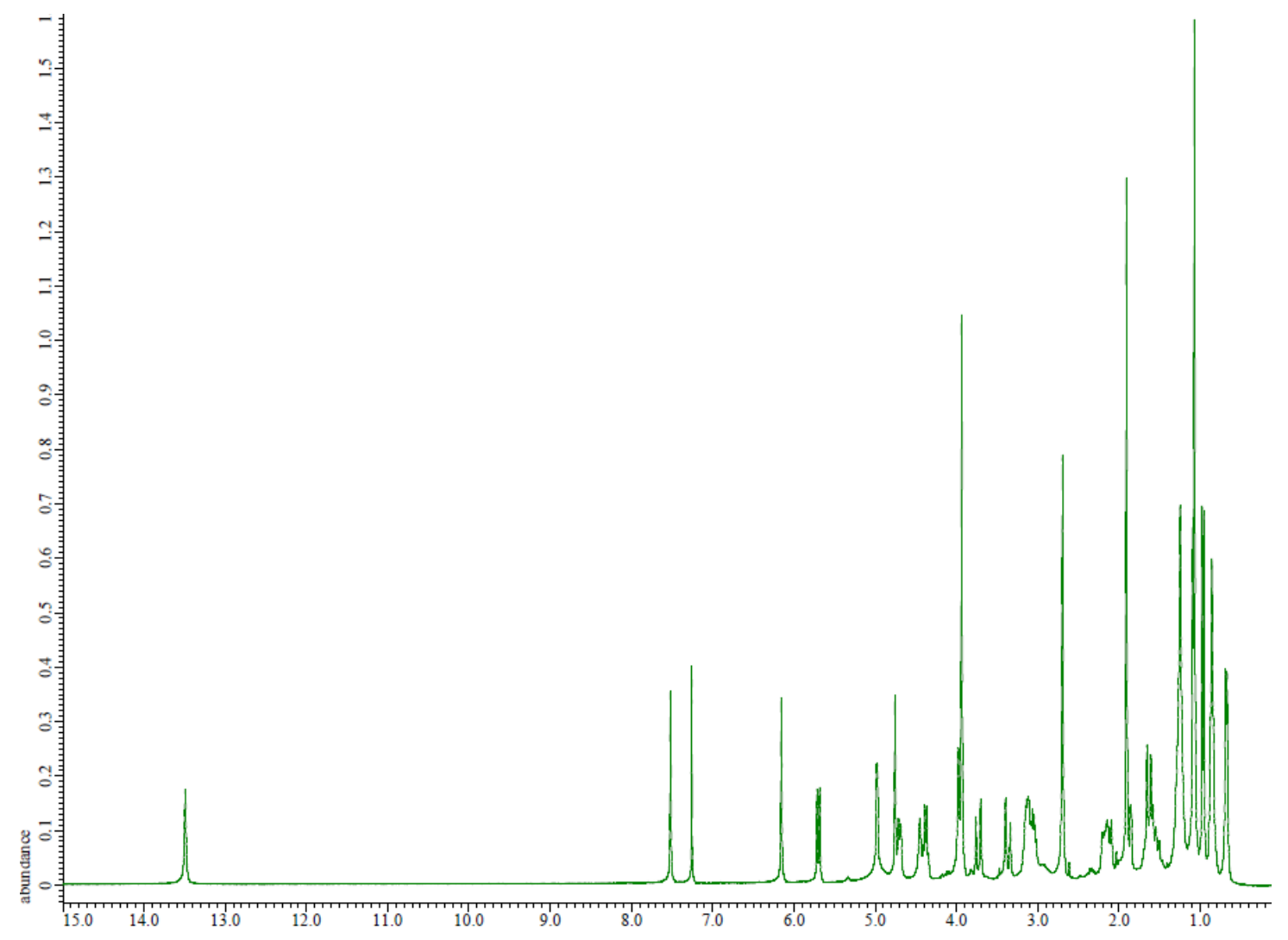

h

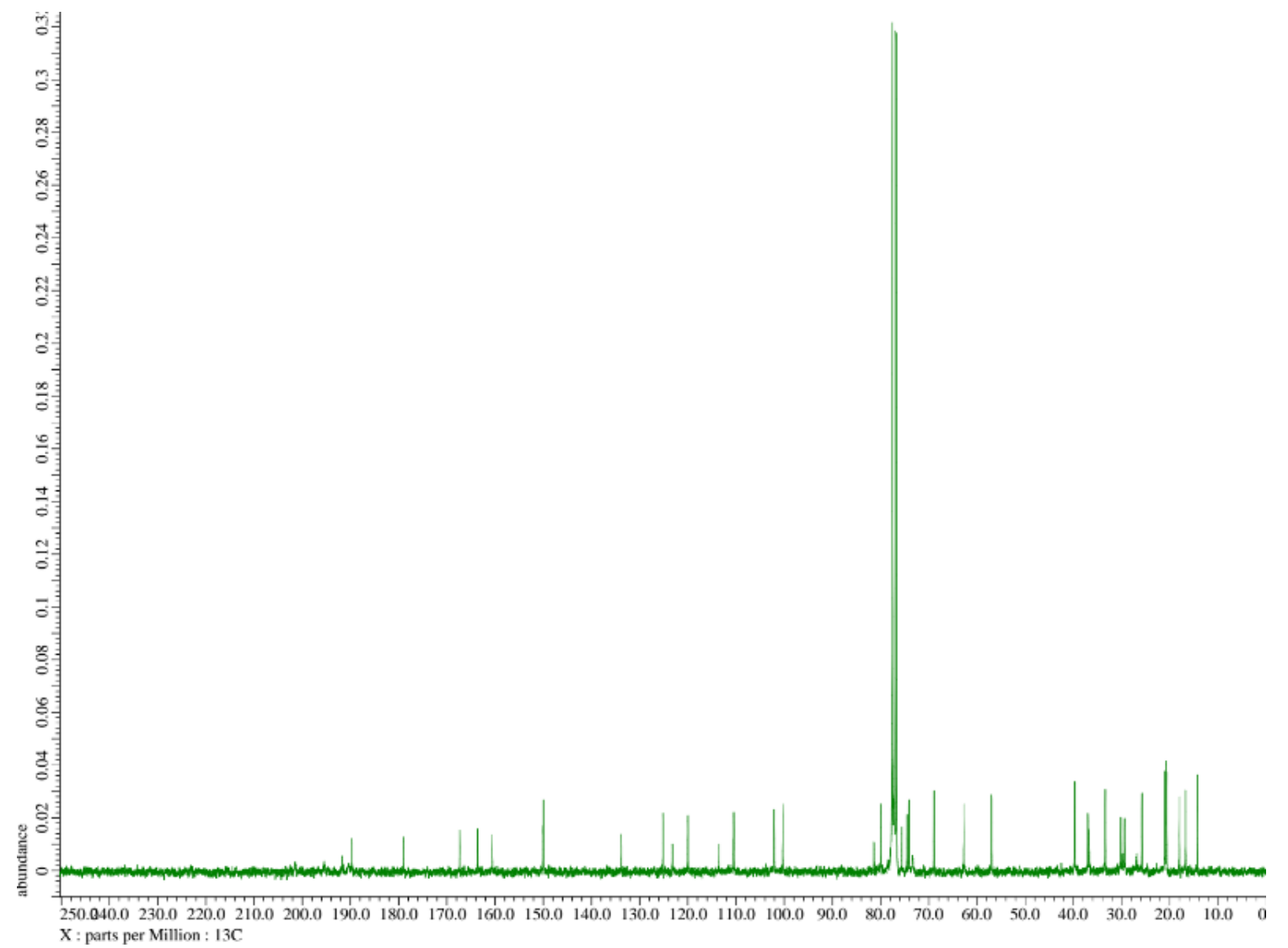


i

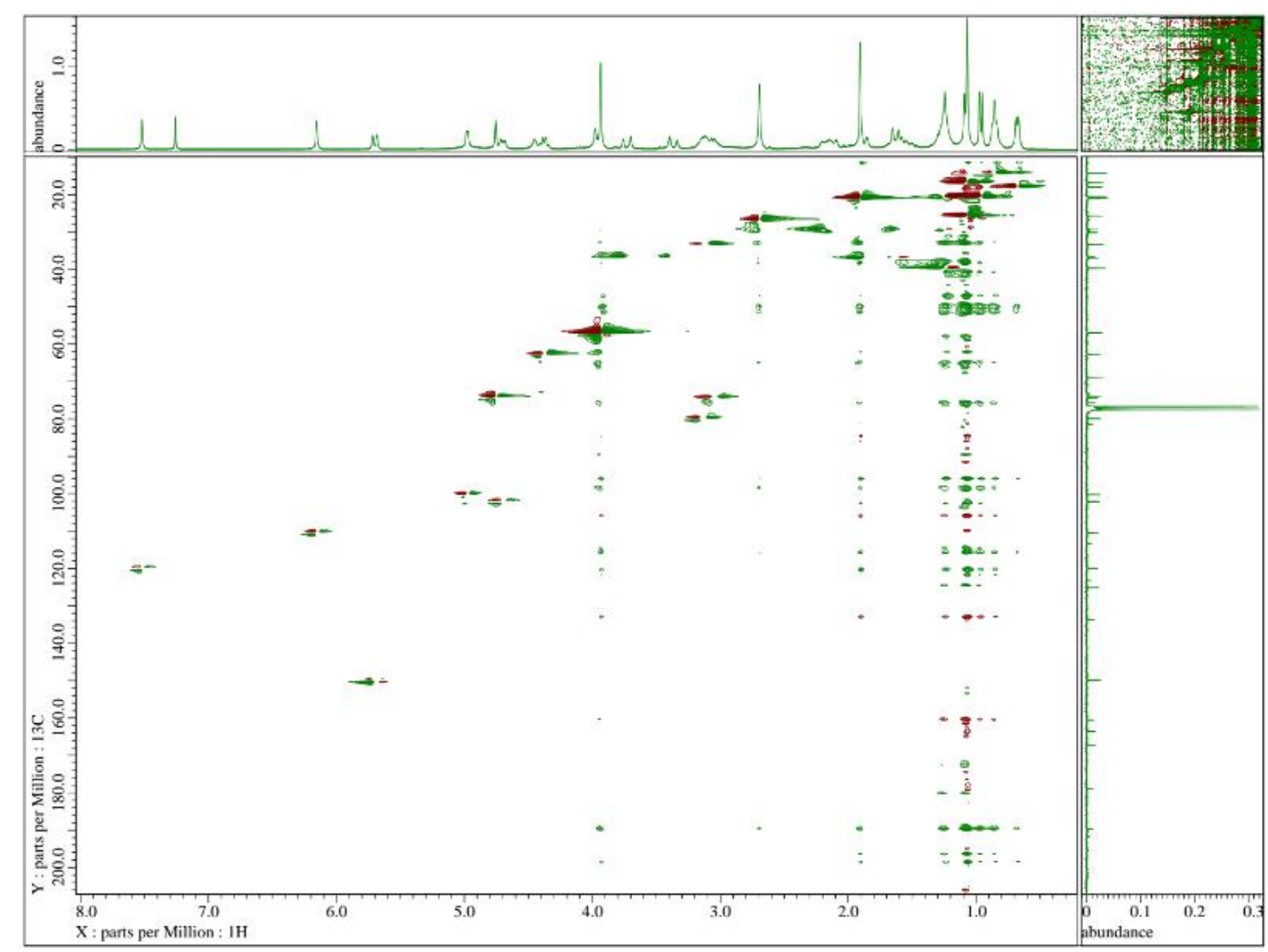

j

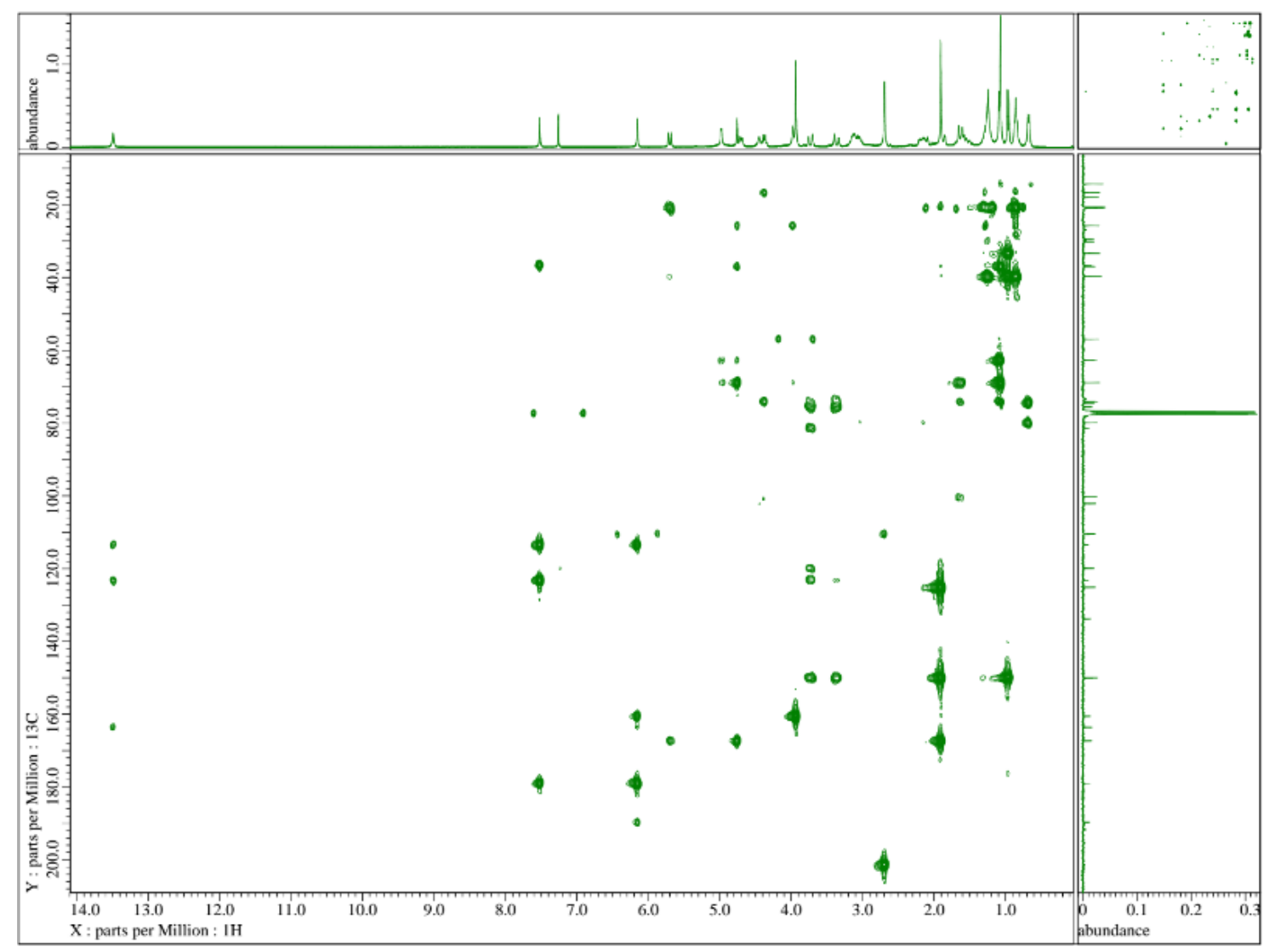


k

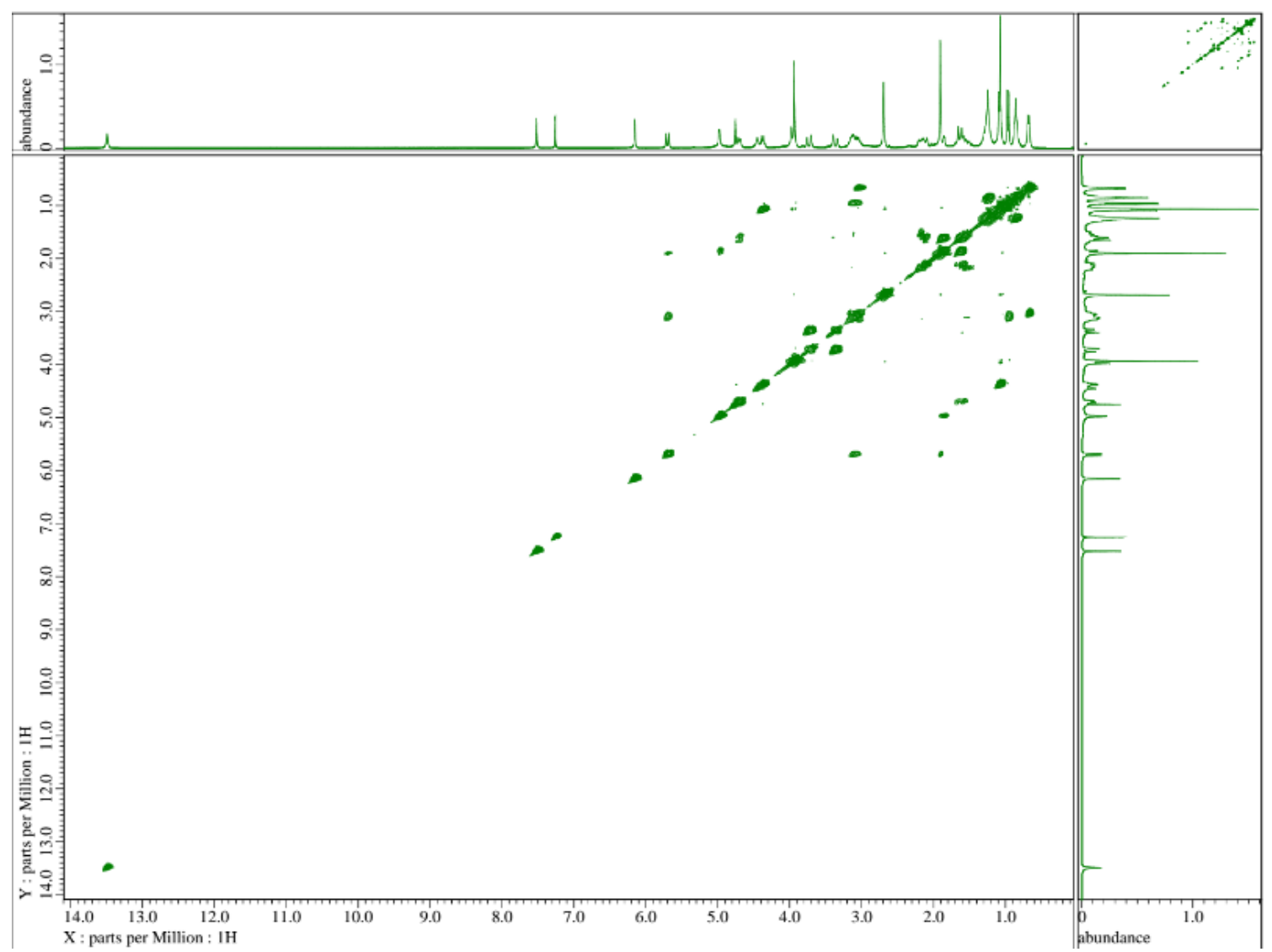

1

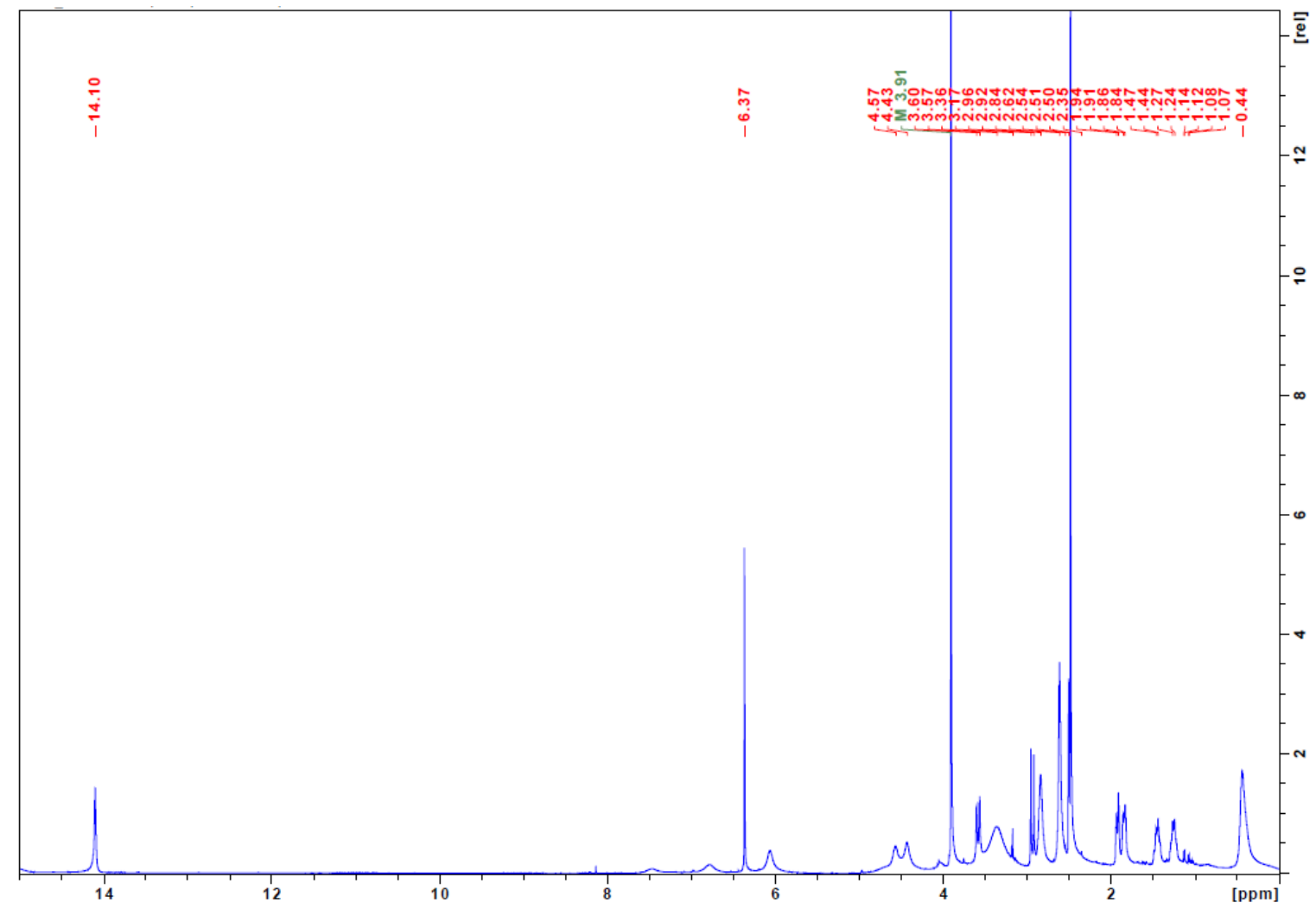




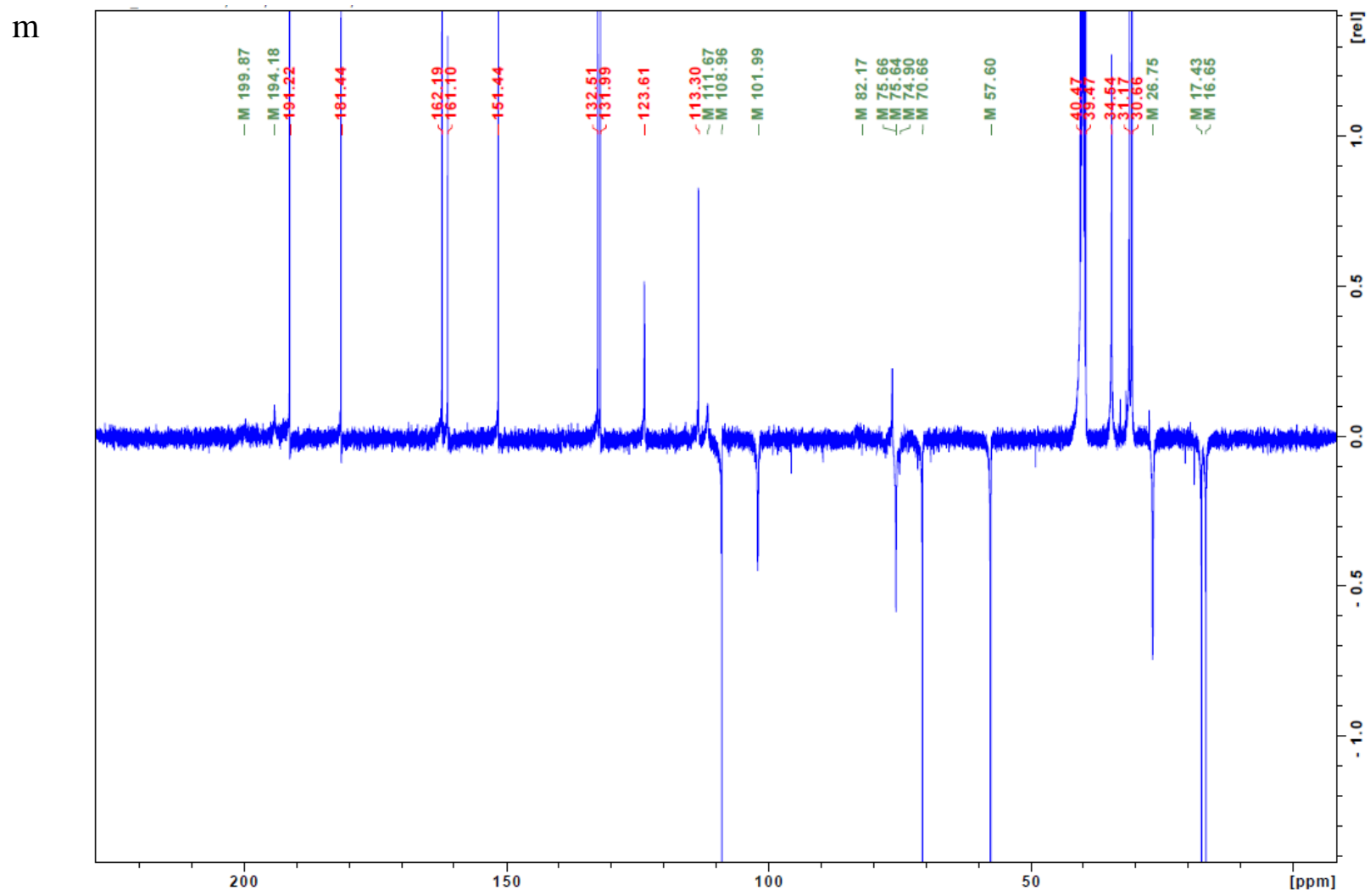

n

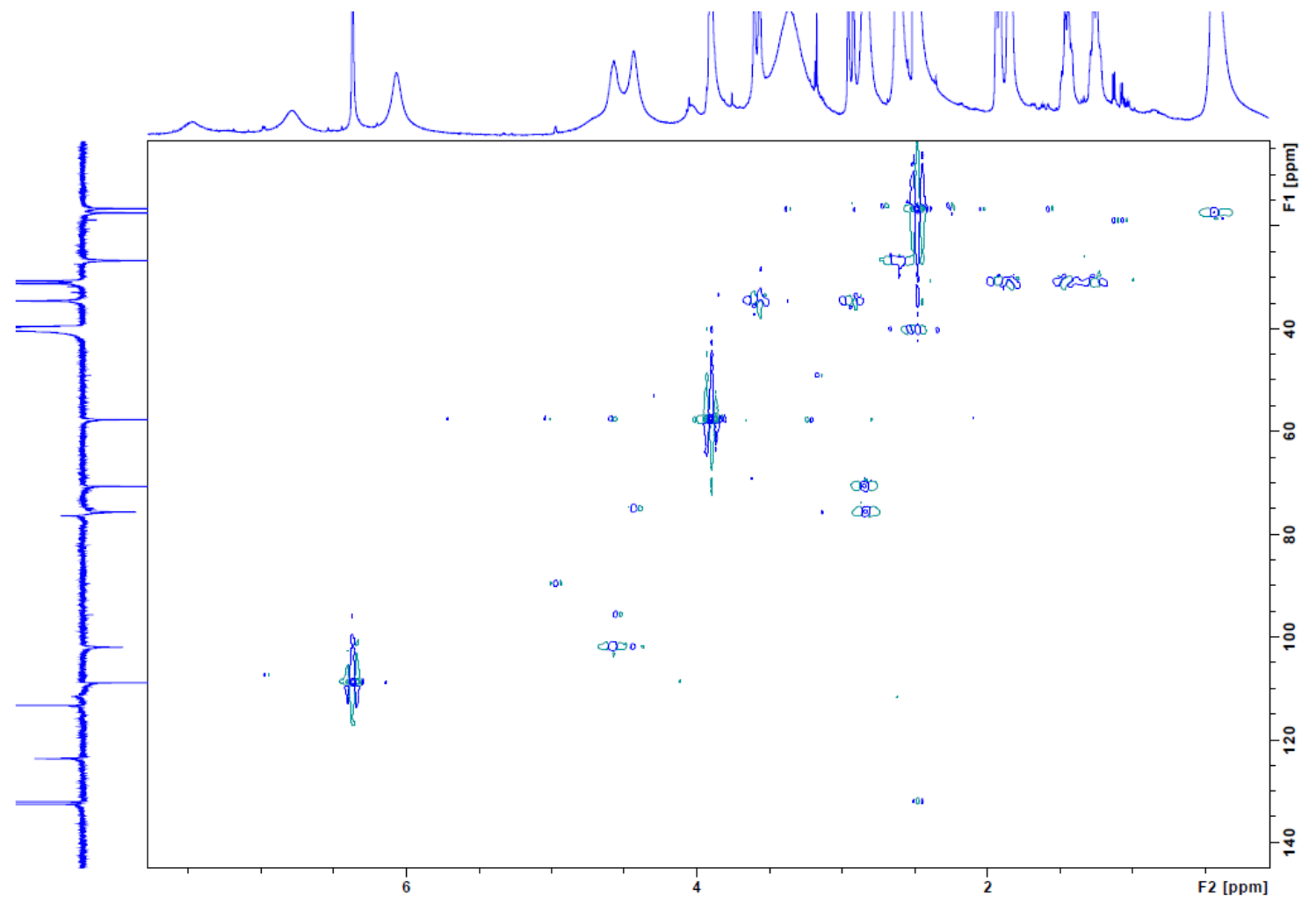


o

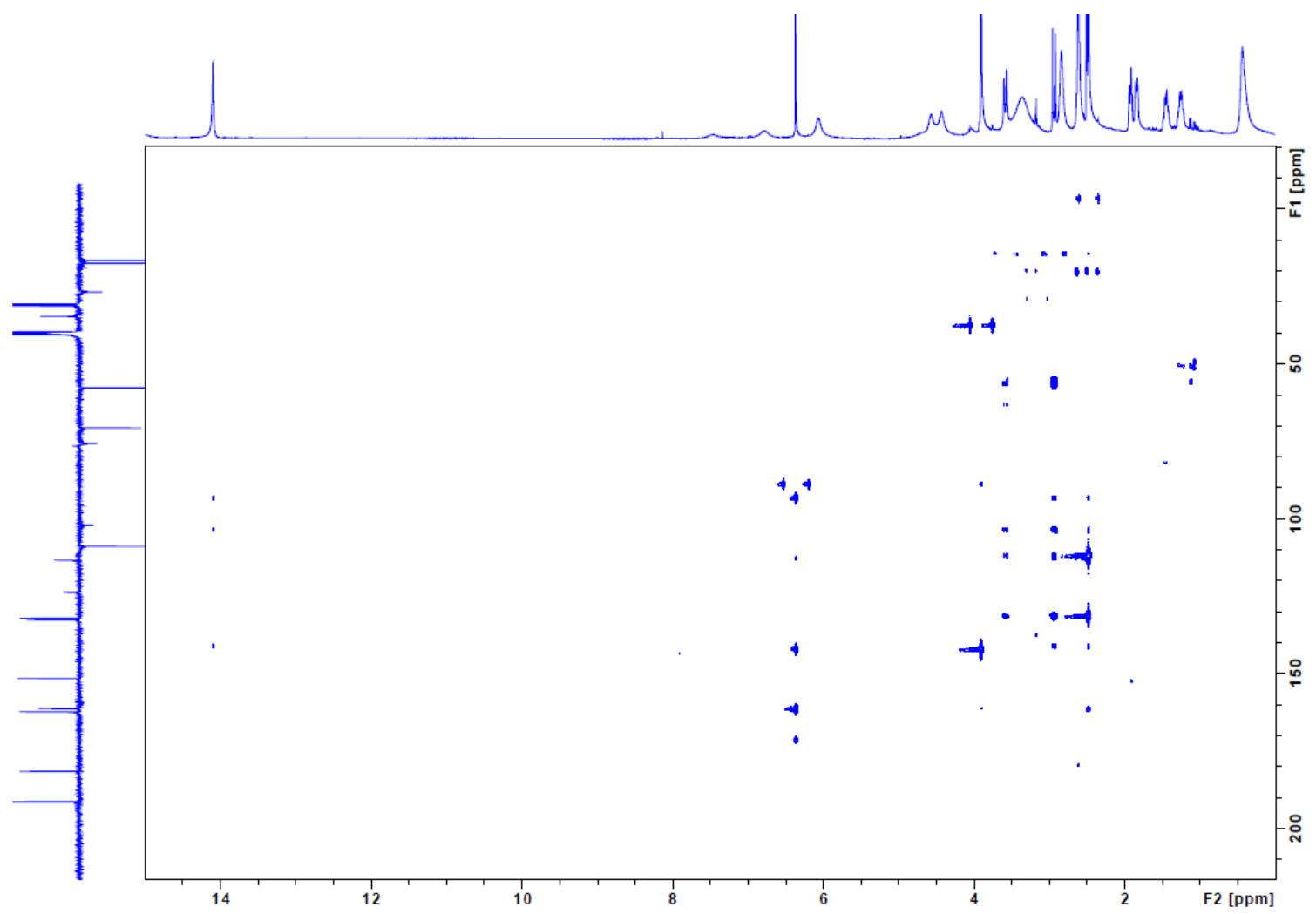

Figure S3. NMR spectra of 1-4. a) ${ }^{1} \mathrm{H}$ NMR spectrum of 1; b) ${ }^{13} \mathrm{C}$ NMR spectrum of $\mathbf{1}$; c) HSQC spectrum of 1 ; d) HMBC spectrum of $\mathbf{1}$; e) ${ }^{1} \mathrm{H}$ NMR spectrum of 2 ; f) ${ }^{13} \mathrm{C}$ NMR spectrum of 2 ; g) ${ }^{1} \mathrm{H}$ NMR spectrum of $\mathbf{3}$; h) ${ }^{13} \mathrm{C}$ NMR spectrum of $\mathbf{3}$; i) HSQC spectrum of $\mathbf{3}$; j) HMBC spectrum of 3 ; k) ${ }^{1} \mathrm{H}-{ }^{1} \mathrm{H}$ COSY spectrum of $\left.3 ; 1\right){ }^{1} \mathrm{H}$ NMR spectrum of $\left.4 ; \mathrm{m}\right){ }^{13} \mathrm{C}$ NMR spectrum of 4 ; $\mathrm{n}$ ) HSQC spectrum of 4; o) HMBC spectrum of 4. 


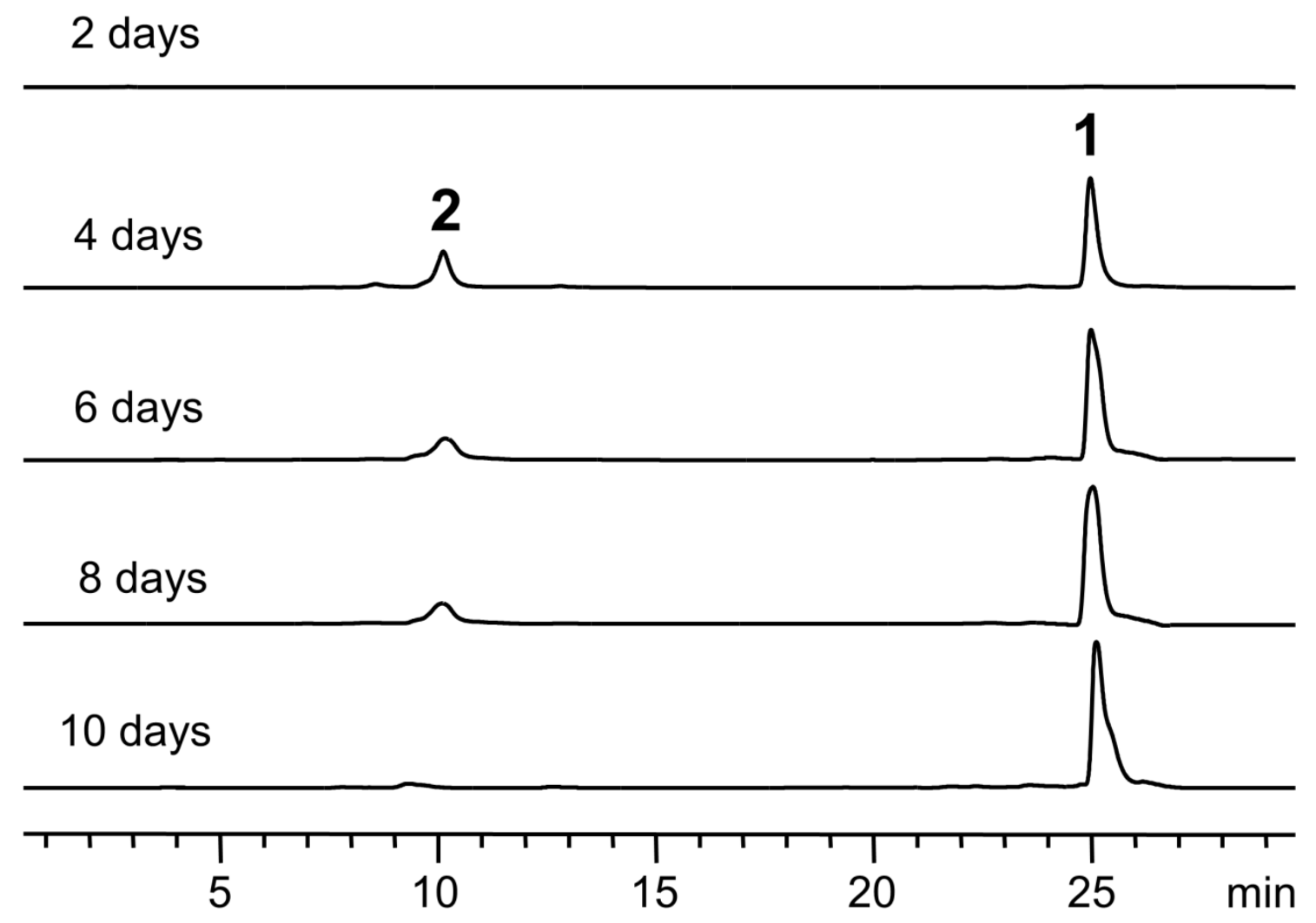

Figure S4. Time course analysis of the production of 1 and $\mathbf{2}$ by S. minoensis NRRL B-5482. 


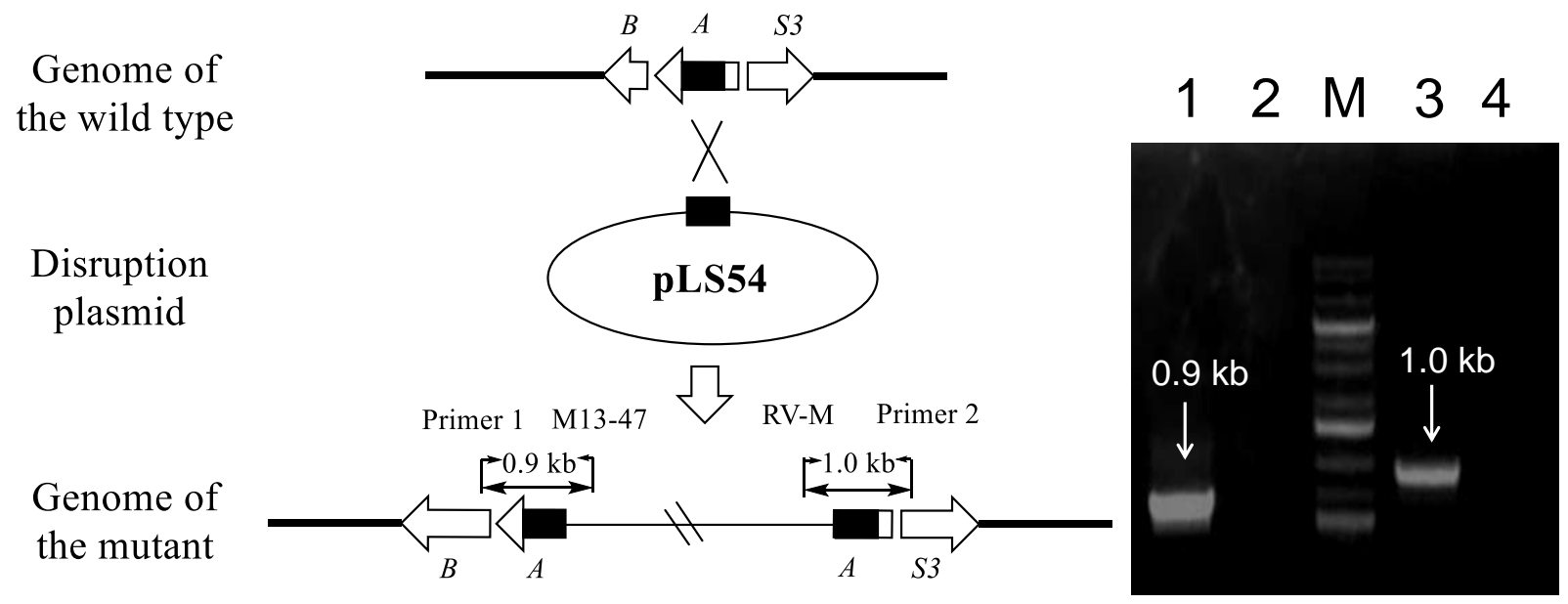

Figure S5. PCR verification of the Streptomyces minoensis NRRL B-5482- $\Delta$ DutA. M: 1-kb Plus DNA ladder; 1: 1.0-kb PCR product from the mutant with primers M13-47 and DutA-Check1; 2: PCR product from the wild type with primers M13-47 and DutA-Check1; 3: PCR product from the wild type with primers RM-V and DutA-Check2; 4: 1.0-kb PCR product from the mutant with primers RM-V and DutA-Check2. 


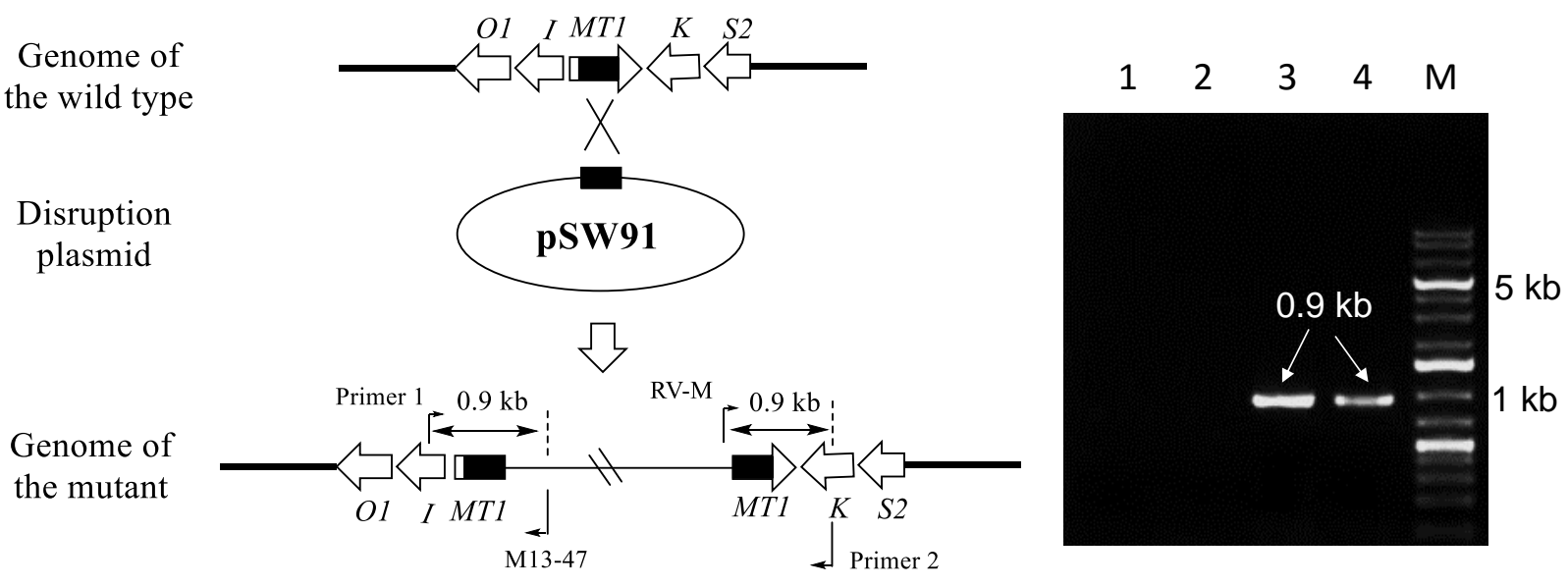

Figure S6. Synthesis of 12-desmethyl-dutomycin (SW91, 3) by disrupting dutMT1. a) Single crossover strategy to disrupt dutMT1, b) PCR verification of the S. minoensis NRRL B-5482$\triangle$ DutMT1 mutant. M: 1-kb Plus DNA ladder; 1: PCR product from the mutant with primers M13-47 and DutMT1-Check1; 2: PCR product from the wild type with primers M13-47 and DutMT1-Check1; 3: PCR product from the mutant with primers RM-V and DutMT1-Check2; 4: PCR product from the wild type with primers RM-V and DutMT1-Check2. 


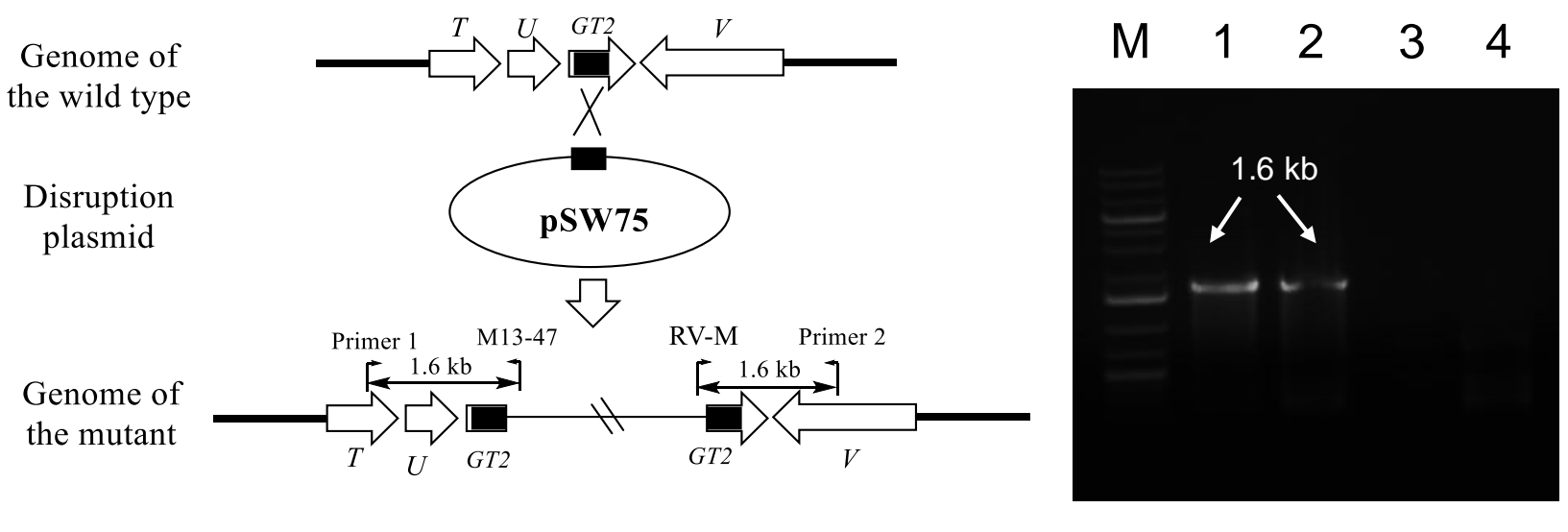

Figure S7. PCR verification of the S. minoensis NRRL B-5482- $\Delta$ DutGT2 mutant. M: 1-kb Plus DNA ladder; 1: 1.6-kb PCR product from the mutant with primers M13-47 and DutGT2-Check1; 2: 1.6-kb PCR product from the mutant with primers RM-V and DutGT2-Check2; 3: PCR product from the wild type with primers M13-47 and DutGT2-Check1; 4: PCR product from the wild type with primers RM-V and DutGT2-Check2. 


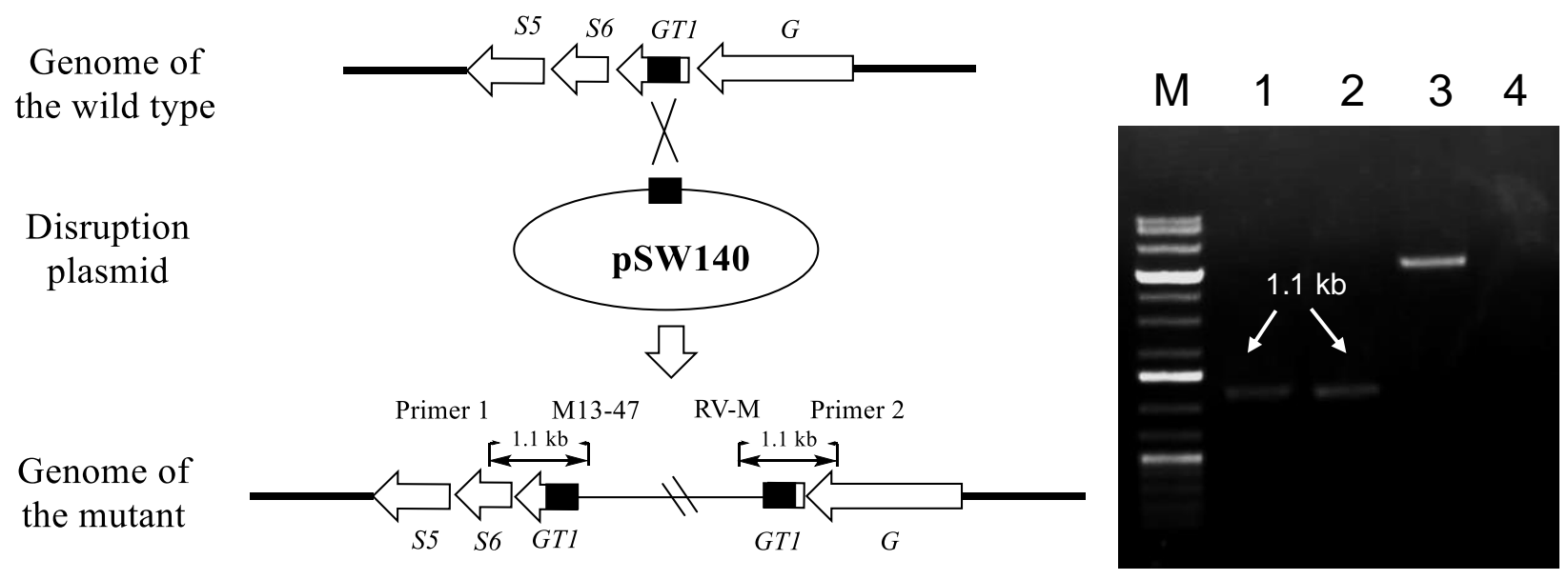

Figure S8. PCR verification of the $S$. minoensis NRRL B-5482- $\Delta$ DutGT1 mutant. M: 1-kb Plus DNA ladder; 1: 1.1-kb PCR product from the mutant with primers M13-47 and DutGT1-Check1; 2: 1.1-kb PCR product from the mutant with primers RM-V and DutGT1-Check2; 3: PCR product from the wild type with primers M13-47 and DutGT1-Check1; 4: PCR product from the wild type with primers RM-V and DutGT1-Check2. 


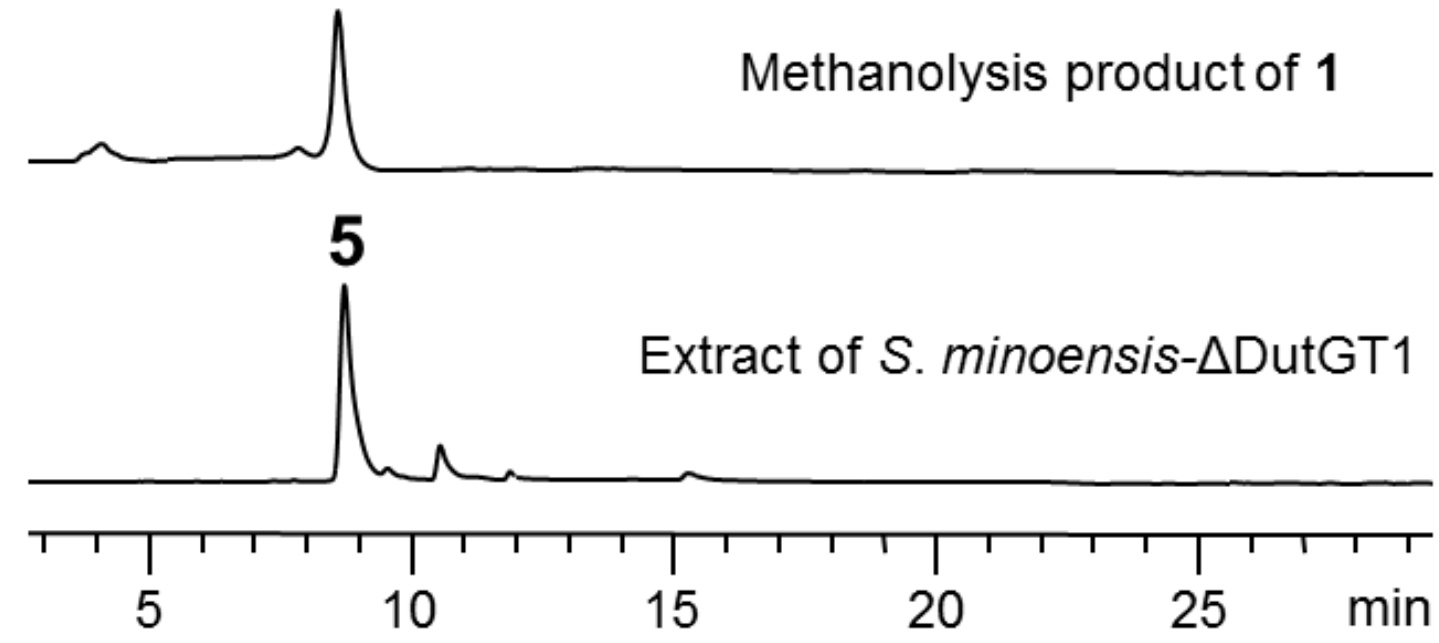

Figure S9. Comparison of the major product (5) of $S$. minoensis- $\Delta$ DutGT1 with the methanolysis product of $\mathbf{1}$ on HPLC at $460 \mathrm{~nm}$. 